\title{
Evolutionary Strategies of Adaptive Plan System with Genetic Algorithm*
}

\author{
Hieu PHAM ${ }^{* *}$, Sousuke TOOYAMA** and Hiroshi HASEGAWA** \\ ** College of Systems Engineering and Science, Shibaura Institute of Technology \\ 307 Fukasaku, Minuma-ku, Saitama-shi, Saitama 337-8570, Japan \\ E-mail: pnh112@gmail.com
}

\begin{abstract}
A new method of Adaptive Plan system with Genetic Algorithm called APGA is proposed to reduce a large amount of calculation cost and to improve a stability in convergence to an optimal solution for multi-peak optimization problems with multidimensions. This is an approach that combines the global search ability of Genetic Algorithm (GA) and the local search ability of Adaptive Plan (AP). The APGA differs from GAs in handling design variable vectors (DVs). GAs generally encode DVs into genes and handle them through GA operators. However, the APGA encodes control variable vectors (CVs) of $\mathrm{AP}$, which searches for local optimum, into its genes. CVs determine the global behavior of AP, and DVs are handled by AP in the optimization process of APGA. In this paper, we introduce some strategies using APGA to solve a huge scale of optimization problem and to improve the convergence towards the optimal solution. These methodologies are applied to several benchmark functions with multi-dimensions to evaluate its performance. We confirmed satisfactory performance through various benchmark tests.
\end{abstract}

Key words : Adaptive System, Genetic Algorithms, Global-Local Search, Memetic Algorithms, Multi-Peak Problems

\section{Introduction}

Product design is becoming more and more complex because of various requirements from customers and their claims. As a consequence, the problems associated with product design seem to be multi-peak problem with multi-dimensions. It is necessary to solve the multi-peak optimization problems. Genetic Algorithm $(\mathrm{GA})^{(1),(2)}$ is the most prominent emergent computing method that has been applied to various multi-peak optimization problems. The validity of this method has been reported by many researchers. Digalakis and Margaritis presented a review and experimental results on major benchmark functions which are used for performance and control of $\mathrm{GA}^{(3)}$. Sakuma and Kobayashi have proposed a real-coded GA using the combination of two crossovers, UNDX-m and $\mathrm{EDX}^{(4)}$, where the effect is reported through benchmark functions. Li and Kirley have introduced fine-grained parallel GA, which is derived from percolation theory ${ }^{(5)}$. Author group has reported the efficiency of GA-based approaches through various studies, including topology optimization for a mechanical structure $^{(6)-(8)}$, the development of a General-purpose Optimization Engine (GOE), and the application of the GOE to various optimal designs ${ }^{(9),(10)}$. However, emergent computing methods require a huge computational cost to obtain stability in the convergence to an optimal solution.

To reduce the cost and to improve stability, a strategy that combines global and local search methods becomes necessary. As for this strategy, current research has proposed various methods. The popular approach is a combination of global search ability of GAs with local search ability of Simulated Annealing $(\mathrm{SA})^{(11)}$. As a pioneering research, Mahfoud and Goldberg have proposed Parallel Recombinative Simulated Annealing (PRSA) that applied SA to a selection of $\mathrm{GA}^{(12)}$. Later, Uehara et al. have introduced metropolis loop process of SA to an

${ }^{*}$ Received 29 Apr., 2012 (No. 12-0198) [DOI: 10.1299/jcst.6.129]

Copyright (c) 2012 by JSME 
elite strategy in GA process ${ }^{(13),(14)}$. Hiroyasu et al. have proposed Parallel SA using Genetic crossover (PSA/ANGA) ${ }^{(15),(16)}$. These hybrid methods have been applied to major benchmark functions and have been reported to be valid. They are believed to be both locally and globally efficient. However, the major multi-peak benchmark functions for multi-dimensions, i.e., 20 dimensional or more Rastrigin (RA) and Griewank (GR) functions, require about $10^{6}$ function calls for arriving at an optimal solution. Moreover, when the optimal problem exhibits a dependence on design variable vectors (DVs) and the steepness of the objective function is small in the feasible space of DVs, it is difficult to obtain an optimal solution ${ }^{(17)}$.

Various optimization methodologies are proposed to overcome these difficulties ${ }^{(17)-(25)}$. In Memetic Algorithms (MAs) ${ }^{(18)-(23)}$, for instance, Ong and Keane has proposed metaLamarckian learning ${ }^{(18)}$ that improves the search ability for multi-peak functions with multidimensions by introducing a human expert judgment, where local search methods are used. Additionally, Fast Adaptive Memetic Algorithm (FAMA) has been proposed in Ref. (22). In the FAMA, coordination and choosing of local search method are dynamically controlled by means of a measurement of fitness diversity over the individuals of the population. On the other hand, Hasegawa et al. have proposed a hybrid meta-heuristic method (HMH) by reflecting recognition of dependence relations among design variables automatically, and have reported the effectiveness of this method ${ }^{(17),(24)}$. The HMH needs to switch from the SA to the intuitive method, direct search using the learning result of the dependency of a DV, just before convergence to improve the local search ability of the optimal solution environs. These methodologies need to choose suitably a best local search method from various local search methods for combining with a global search method within the optimization process. Furthermore, since genetic operators are employed for a global search method within these algorithms, DVs which are renewed via a local search are encoded into its genes many times at its GA process. These certainly have the potential to break its improved chromosomes via gene manipulation by GA operators, even if these approaches choose a proper survival strategy.

Hasegawa has proposed a new Evolutionary Algorithm (EA) called an Adaptive Plan System with Genetic Algorithm (APGA) ${ }^{(25)}$ to solve these problems and maintain the stability of the convergence to an optimal solution for multi-peak optimization problems with multidimensions. In this paper, we introduce some strategies using APGA to dramatically improve the stability up to the optimal solution.

This paper is organized in the following manner. The algorithm of proposed strategy, APGA is described in Section 2, the concept of Variable Neighborhood range Control (VNC) is in Section 3. Section 4 explains the hybrid neighborhood control method of APGA (HAPGA), and Section 5 discusses about the convergence to the optimal solution of multi-peak benchmark functions. Finally, Section 6 includes some brief conclusions and future works.

\section{The Proposed Strategy - APGA}

\subsection{Formulation of Optimization Problem}

The optimization problem is formulated in this section. Design variable vectors (DVs), objective function and range of DVs are defined as follow:

Design variable: $\quad X=\left[x_{1}, \ldots, x_{n}\right]$

Objective function : $\quad-f(X) \rightarrow$ Max

Range of DVs:

$$
X^{L B} \leq X \leq X^{U B}
$$

Where $X^{L B}=\left[x_{1}^{L B}, \ldots, x_{n}^{L B}\right], X^{U B}=\left[x_{1}^{U B}, \ldots, x_{n}^{U B}\right]$, and $n$ denote the lower boundary condition vectors, the upper boundary condition vectors, and the number of DVs, respectively. A number of DVs significant figure is defined, and DV is rounded off to its decimal places within optimization process. 


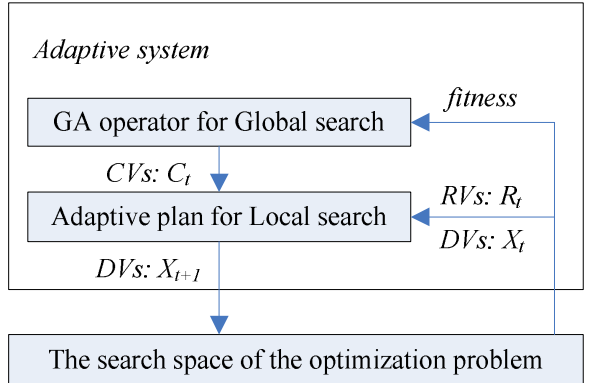

Fig. 1 APGA conceptual process

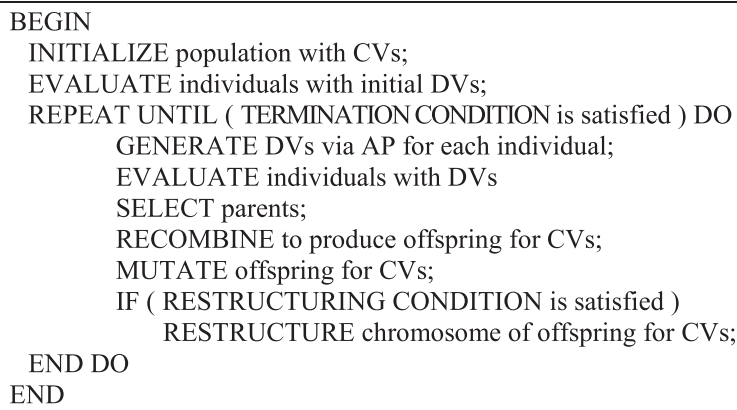

Fig. 2 APGA Algorithm

\subsection{APGA}

Natural and artificial systems adapt their behavior to changes in global and social environments over generations. Holland has defined this type of system as an Adaptive System $(\mathrm{AS})^{(1)}$, and the AS has an Adaptive Plan (AP) that determines its behaviors through responses to environments.

The APGA concept was introduced as a new EA strategy for multi-peak optimization problems. Its concept differs in handling DVs from general EAs based on GAs. EAs generally encode DVs into the genes of a chromosome, and handle them through GA operators. However, APGA completely separates DVs of global search and local search methods. It encodes Control variable vectors (CVs) of AP, which searches for local minima, into its genes on AS. Moreover, this separation strategy for DVs and chromosomes can solve MA problem of breaking chromosomes.

The conceptual process of APGA is shown in Fig. 1. In the APGA, the design space of the optimization problems, DVs, and response value vectors (RVs) are defined as the environment of AS, the point of the design space and response values from its environment, respectively. The CVs steer the individuals global behavior of AP for a global search, and are renewed via genetic operations by estimating fitness value which is a part of RVs. For a local search, AP generates next values of DVs by using CVs, RVs, and current values of DVs in the APGA optimization process. The DV generation process generates a new DV from the current search point via AP according to the formula:

$$
X_{t+1}=X_{t}+N R_{t} \cdot A P\left(C_{t}, R_{t}\right)
$$

Where $N R, A P(), X, C, R$, and $t$ denote neighborhood ratio, a function of AP, DVs, CVs, $\mathrm{RV}$ s and generation, respectively.

The APGA's algorithm is described by the pseudocode given in Fig. 2. In addition, for a verification of APGA search process, refer to Ref. (17).

\subsection{Adaptive Plan - Sensitivity Plan}

It is necessary that the AP realizes a local search process by applying various heuristics rules. In this paper, the plan introduces a DV generation formula using a sensitivity analy- 


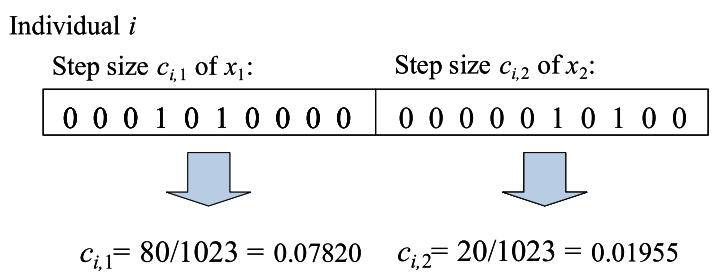

Fig. 3 Encoding into genes of a chromosome

sis that is effective in the convex function problem as a heuristic rule, because a multi-peak problem is combined of convex functions. This plan uses the following equation:

$$
\begin{aligned}
& A P\left(C_{t}, R_{t}\right)=- \text { Scale } \cdot S P \cdot \operatorname{sign}\left(\nabla R_{t}\right) \\
& S P=2 C_{t}-1
\end{aligned}
$$

Where $S$ cale, and $\nabla R$ denote scale factor and sensitivity of RVs, respectively.

A step size $S P$ is defined by $\mathrm{CV}$ s for controlling a global behavior to prevent it falling into the local optimum. $C=\left[c_{i, j}, \ldots, c_{i, p}\right] ;\left(0.0 \leq c_{i, j} \leq 1.0\right)$ is used by eq. (6) so that it can change the direction to improve or worsen the objective function, and $C$ is encoded into a chromosome by 10 bit strings (shown in Fig. 3). In addition, $i, j$ and $p$ are the individual number, design variable number and its size, respectively.

2.3.1. Handling of Significant Figures In the optimal design of the product design, dimensions of products can be mainly dealt with as DVs. There are always assigned dimensional accuracies on a mechanical drawing. Therefore, a value of DVs is done well to use a number of significant figures of assigned dimensional accuracy in its drawing in the optimal process. In APGA, a number of significant figures of DVs are defined, and DVs are truncated to it within optimal process.

2.3.2. Handling out of Range DVs DVs are renewed by the AP, and when their values exceed their range, the APGA returns them to their range by using equation:

$$
\begin{cases}X_{t}=2 X^{L B}-X_{t}, & X_{t}<X^{L B} \\ X_{t}=2 X^{U B}-X_{t}, & X_{t}>X^{U B}\end{cases}
$$

2.3.3. Coding into Chromosome for CVs CVs are individually coded into a string to form a chromosome. This 10 bit string with two values ( 0 and 1$)$ represents a real value of $\mathrm{CV}$ s by using the procedure shown in Fig. 3. In addition, this figure shows both DVs and CVs of 2 dimensions cases.

\subsection{Control of Neighborhood Range}

In case, the optimal problem of benchmark function exhibits a dependency on DVs and the steepness of this function is small, to solve its multiple dimensional optimization problem is difficulty.

For overcoming this difficulty, we propose the control of neighborhood range. Applying a neighborhood range control method is a common approach for an SA, and the distance for a search point can be changed by controlling the neighborhood range. The neighborhood range is decided by neighborhood ratio, according to the formula, as in:

$$
N R_{t}=\left(t_{\max }-t\right) /\left(t_{\max }-1\right)
$$

Where $t_{\max }$ denote terminal generation. Equation (8) is a linear formula, and the relationship between the neighborhood ratio and the number of generation is shown in Fig. 4.

The neighborhood range of the early generations is adjusted to wide range by the control using this relationship. Moreover, it is modified to small range at the late generations. As results, we believe that search process is varied according to this control schedule roughly or delicately toward the good search direction. To increase search distance of early generations, scale factors are used in eq. (5). 


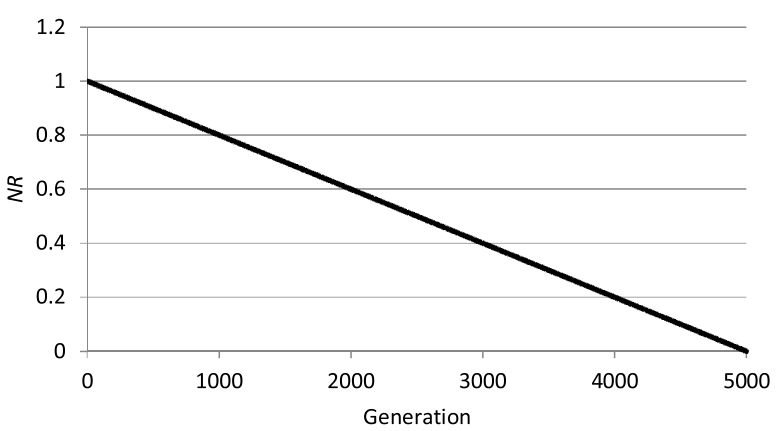

Fig. 4 Relationship between neighborhood ratio and generation

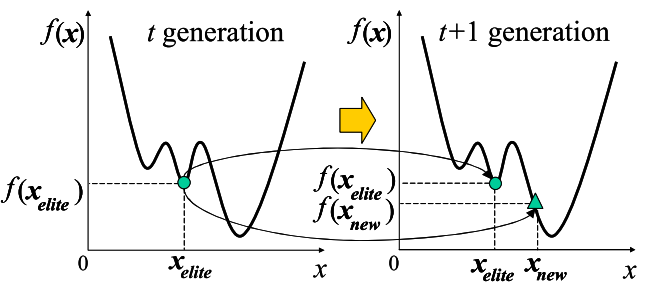

Fig. 5 Elite strategy

\subsection{GA Operators}

2.5.1. Selection Selection is performed using a tournament strategy to maintain the diverseness of individuals with a goal of keeping off an early convergence. A tournament size of 2 is used.

2.5.2. Elite Strategy An elite strategy, where the best individual survives in the next generation, is adopted during each generation process.

It is necessary to assume that the best individual, i.e., as for the elite individual, generates two behaviors of AP by updating DVs with AP, not GA operators. Therefore, its strategy replicates the best individual to two elite individuals, and keeps them to next generation. As shown in Fig. 5, DVs of one of them ( $\Delta$ symbol) is renewed by AP, and its CVs which are coded into chromosome are not changed by GA operators. Another one (o symbol) is that both DVs and CVs are not renewed, and are kept to next generation as an elite individual at the same search point.

2.5.3. Crossover and Mutation In order to pick up the best values of each $\mathrm{CV}$, a single point crossover is used for the string of each $\mathrm{CV}$. This can be considered to be a uniform crossover for the string of the chromosome as shown in Fig. 6(a).

Mutation are performed for each string at mutation ratio on each generation, and set to maintain the strings diverse as shown in Fig. 6(b).

2.5.4. Recombination of Genes At following conditions, the genetic information on chromosome of individual is recombined by uniform random function.

(1) One fitness value occupies $80 \%$ of the fitness of all individuals

(2) One chromosome occupies $80 \%$ of the population

If this manipulation is applied to general GAs, an improved chromosome into which DVs have been encoded is broken down. However, in the APGA, the genetic information is only CVs used to make a decision for the AP behavior. Therefore, to prevent from falling into a local optimum, and to get out from the condition of being converged with a local optimum, a new AP behavior is provided by recombining the genes of the CVs into a chromosome. And the optimal search process starts to re-explore by a new one. This strategy is believed to make behavior like the re-annealing of an SA.

\section{Variable Neighborhood Range Control - VNC}

In the multi-point search of the APGA, individuals move from their various points to new search points in the design space of DVs. For example, as shown in Fig. 7, individual 


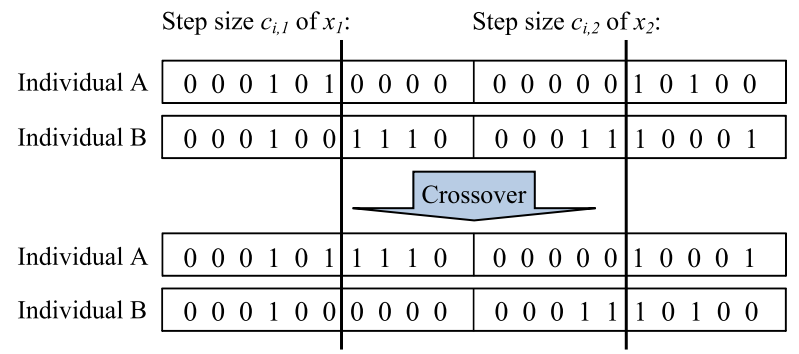

(a)

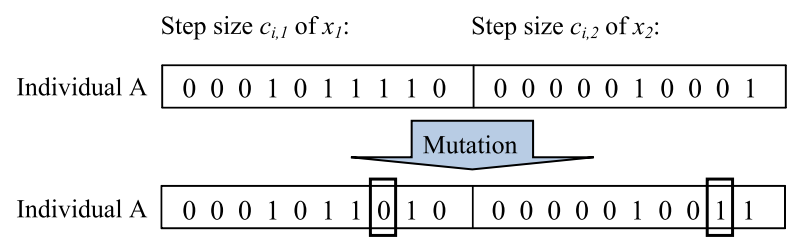

(b)

Fig. 6 Crossover and Mutation

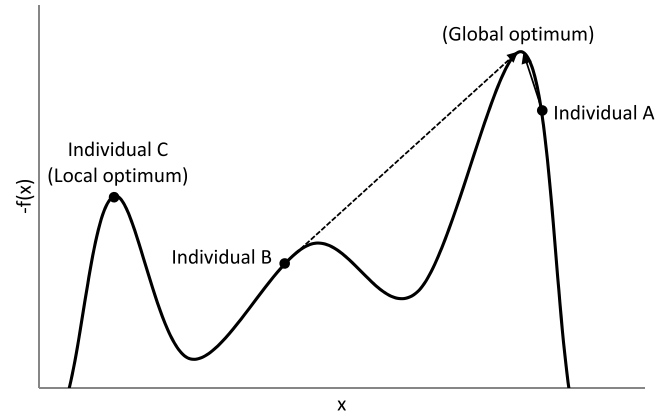

Fig. 7 Example of individual situations

A requires a slight change to the values of the DVs to obtain the global optimum solution. On the other hand, individual B cannot reach a global optimum solution without a significant change. In addition, individual $\mathrm{C}$ has landed at a local optimum solution. Such a situation, in which the individuals are intermingled, can generally occur at any time in search process. Therefore, it is necessary to find a suitable DV generation process for the situation of each individual in the design space.

To improve the multi-point search capability of the APGA, we propose a Variable Neighborhood Range Control (VNC) method, introduced to the AS. This method automatically adapts the neighborhood range to obtain DV generation accuracy for the situation of each individual. As a result, we believe that it will steadily provide a global optimum solution and reduce the calculation cost.

\subsection{Assignation of Variable Neighborhood Range}

In the VNC method, APGA process generates a new DV via AP using a sensitivity analysis by eq. (4).

The variable neighborhood range is determined by the $N R$, and the assignation step for the NR is shown in Fig. 8. The VNC method sorts all of the individuals by estimating their fitness, then ranking them by results. A ranked individual is labeled with the rank number and is assigned an $N R$ that corresponds with this number. In this method, the formula for the $N R$ by sigmoid function is as follows:

$$
N R_{t}=\frac{1}{1+\exp \left(\alpha \cdot \frac{\text { rank-(invidual } / 2)}{\text { invidual }}\right)}
$$




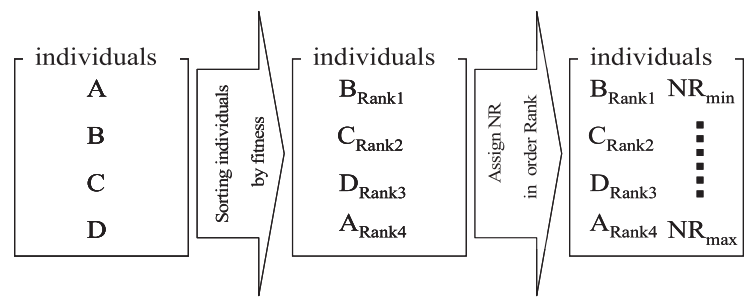

Fig. 8 Assignation step for neighborhood range

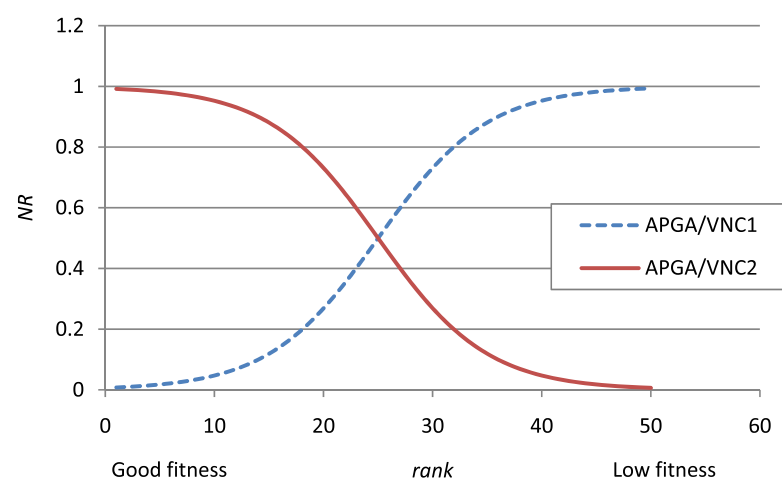

Fig. 9 Variable Neighborhood Control by APGA/VNC $(|\alpha|=10.0)$. Note that, if the absolute value of gain $\alpha$ is increased, the gradient of the sigmoid function becomes steep

$0.0 \leq N R_{t} \leq 1.0$

Where $\alpha$, rank, and individual denote the gain of the sigmoid function, rank number and number of individual, respectively.

The search process is varied according to these models, roughly or delicately, toward the best search direction for all of individuals.

\subsection{APGA/VNC Method}

The APGA/VNC method defines two models by the sign of the gain $\alpha$ as follows:

$\begin{cases}A P G A / V N C 1, & \alpha<0 \\ A P G A / V N C 2, & \alpha>0\end{cases}$

3.2.1. APGA/VNC1 In APGA/VNC1, as shown in Fig. 9, the individuals with high fitness values (good individuals) are allocated small $N R$ values to perform a local search efficiently. On the other hand, the individuals with low fitness values (low individuals) are allocated large $N R$ values to search the global area in the design space of the DVs.

3.2.2. APGA/VNC2 As for the APGA/VNC1, if a good individuals position belongs to a local optimum neighborhood area, the good individual searches its own neighborhood area without performing a global search. Therefore, it cannot escape from the local optimum solution. As a counter-plan for this problem, APGA/VNC2 employs the inverse model of APGA/VNC1 (shown in Fig. 9). This model assigns a large $N R$ to the good individuals. As a result, they cannot search their own neighborhood areas. Moreover, as for the low individuals, this model assigns a small $N R$. Just before converging to the global optimum solution, to allow individuals to gather in the neighborhood area of the elite individual, the low individuals perform a local search.

\section{Hybrid Neighborhood Control Method - H-APGA}

To improve the multi-point search capability of APGA, we propose a Hybrid neighborhood control method introduce into APGA (H-APGA). In H-APGA method, the neighborhood ratio is controlled by mixing between linear function and sigmoid function. 


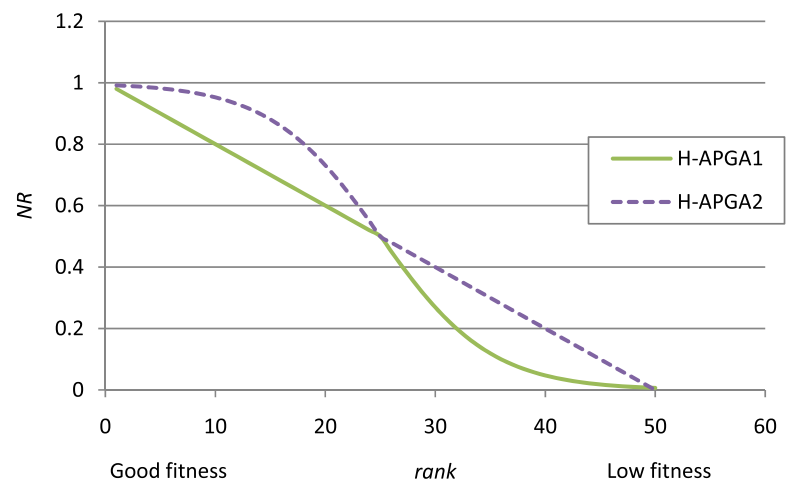

Fig. 10 Neighborhood range control by H-APGA $(\alpha=10.0)$

\subsection{Linear Function}

The neighborhood ratio is determined by $N R$, and the formula for the $N R$ by linear function is as follows:

$$
N R_{t}=1-\frac{1}{2}\left(\frac{i n v}{\text { invidual } / 2}\right)
$$

Where inv, individual denote the current individual and number of individual.

To calculate $N R$, this method uses individual number only. Therefore, $N R$ is distributed to an individual at random. It is adjusted to wire range with the first half of individual number and it is modified to small range with the last haft of one (as shown in Fig. 10).

\subsection{H-APGA Method}

4.2.1. H-APGA1 In H-APGA1, as shown in Fig. 10, the good individuals (high fitness values) are allocated large $N R$ values to search global area in the design space of the DVs following eq. (12). On the other hand, the low individuals (low fitness values) are allocated small $N R$ values to perform a local search efficiently following eq. (9).

4.2.2. H-APGA2 H-APGA2 employs the inverse method of H-APGA1 (as shown in Fig. 10). H-APGA2 assigns a large $N R$ to the good individuals following eq. (9). Moreover, as for the low individuals, this method assigns a small $N R$ following eq. (12). Before converging to the global optimum, the low individuals perform a local search.

\section{Numerical Experiments}

In this section, the numerical experiments are performed to compare among methods. Next, these methods are compared with other methodologies for the robustness of the optimization process. These experiments are performed 20 trials for each function. The initial seed number is randomly varied during every trial. In each experiment, the GA parameters used in solving benchmark functions are set as follows, selection ratio, crossover ratio and mutation ratio are 1.0, 0.8 and 0.01 respectively. The population size is 50 individuals and the terminal generation is $5000^{\text {th }}$ generation.

\subsection{Benchmark Functions}

For the APGAs, we estimate the stability of the convergence to the optimal solution by using five benchmark functions with multiple dimensions Rastrigin (RA), Ridge (RI), Griewank (GR), Rosenbrock (RO) and Ackley (AC) function. These functions are given as follows:

$$
\begin{aligned}
& R A: f_{1}=10 n+\sum_{i=1}^{n}\left\{x_{i}^{2}-10 \cos \left(2 \pi x_{i}\right)\right\} \\
& R I: f_{2}=\sum_{i=1}^{n}\left(\sum_{j=1}^{i} x_{j}\right)^{2}
\end{aligned}
$$


Table 1 Characteristics of benchmark functions

\begin{tabular}{|c|c|c|c|}
\hline Function & Epistasis & Multi-peaks & Steepness \\
\hline RA & No & Yes & Average \\
\hline RI & Yes & No & Average \\
\hline GR & Yes & Yes & Small \\
\hline RO & Yes & No & Big \\
\hline AC & No & Yes & Average \\
\hline
\end{tabular}

Table 2 Range, digits of DVs, and scale factor for normalizing benchmark functions

\begin{tabular}{|c|c|c|c|}
\hline Function & Design range & Number of digits & Scale factor \\
\hline RA & $-5.12 \leq X \leq 5.12$ & 2 & 10.0 \\
\hline RI & $-51.2 \leq X \leq 51.2$ & 1 & 100.0 \\
\hline GR & $-51.2 \leq X \leq 51.2$ & 1 & 100.0 \\
\hline RO & $-2.048 \leq X \leq 2.048$ & 3 & 4.0 \\
\hline AC & $-5.12 \leq X \leq 5.12$ & 2 & 10.0 \\
\hline
\end{tabular}

$$
\begin{aligned}
& G R: f_{3}=1+\sum_{i=1}^{n} \frac{x_{i}^{2}}{4000}-\prod_{i=1}^{n} \cos \left(\frac{x_{i}}{\sqrt{i}}\right) \\
& R O: f_{4}=\sum_{i=1}^{n}\left[100\left(x_{i+1}+1-\left(x_{i}+1\right)^{2}\right)^{2}+x_{i}^{2}\right] \\
& A C: f_{5}=-20 \exp \left(-0.2 \sqrt{\frac{1}{n} \sum_{i=1}^{n} x_{i}^{2}}\right)-\exp \left(\frac{1}{n} \sum_{i=1}^{n} \cos \left(2 \pi x_{i}\right)\right)+20+e
\end{aligned}
$$

Table 1 shows their characteristics, and the terms epistasis, multi-peaks, steepness denote the dependence relation of the DVs, presence of multi-peak and level of steepness, respectively. In Table 2, their design range, the digits of DVs, and the sensitivity plan parameters in eq. (5) are summarized. Note that, it is difficult to search for their optimal solutions by applying one optimization strategy only, because each function has specific complex characteristics.

All functions are minimized to zero, when optimal DVs $X=0$ are obtained. The search process is terminated when the search point attains an optimal solution or a current generation process reaches the termination generation.

\subsection{Experiment Results}

The experiment results are shown in Table 3-7. When the success rate of optimal solution is not $100 \%$," -" is described, and the improvement rate denotes a rate of a calculation cost of APGA/VNC method and H-APGA method compared with simple APGA. The improvement rate is average value of improvement rate of five benchmark functions expect for functions with which the optimal solution was not reached.

The solutions of all benchmark functions with 20 dimensions reach their global optimum solution. However, there are some differences among methods. APGA/VNC1 could not reach a global optimum solution as for some trials of RI and RO function. On the other hand, APGA/VNC2 could arrive at a global optimum solution in all trials. Moreover, H-APGA method converged faster than APGA/VNC method, especially H-APGA2 could arrive at a global optimum at a high probability with every function.

As a result, we assign the best trial that is found by the maximum improvement rate. Its best gain value is trial 2 with APGA/VNC1, trial 9 with APGA/VNC2, trial 19 with H-APGA1 and trial 17 with H-APGA2. Moreover, the results using the best trial of these methods are compared. From this comparison, we can confirm that H-APGA2 is the best solution.

Next, Fig. 11-15 shows trend diagrams for the average fitness of an elite individual until these methods reach global optimum solutions, in the numerical experiment again to confirm above mentioned result.

In summary, its validity confirms that this strategy can reduce the computation cost and improve the stability of the convergence to the optimal solution. 
Table 3 Experiment results, averaged over 20 runs by APGA ( 20 dimensions; population size 50). "Mean" indicates average of optimum values obtained and "Std Dev" stands for standard deviation

\begin{tabular}{|c|c|c|}
\hline Function & Num. of. Gen. & Mean (Std Dev) \\
\hline RA & 376 & $0.00 \mathrm{E}+00(0.00 \mathrm{E}+00)$ \\
\hline RI & 2030 & $0.00 \mathrm{E}+00(0.00 \mathrm{E}+00)$ \\
\hline GR & 468 & $0.00 \mathrm{E}+00(0.00 \mathrm{E}+00)$ \\
\hline RO & - & $-4.78 \mathrm{E}+02(5.14 \mathrm{E}+02)$ \\
\hline AC & 378 & $-4.44 \mathrm{E}-16(0.00 \mathrm{E}+00)$ \\
\hline
\end{tabular}

Table 4 Experiment results by APGA/VNC1. Number of generations required to reach the global optimum (20 dimensions; population size 50)

\begin{tabular}{|c|c|c|c|c|c|c|}
\hline \multirow{2}{*}{ Trial } & \multicolumn{5}{|c|}{ Function } & \multirow{2}{*}{$\begin{array}{c}\text { Improvement } \\
\text { Rate }\end{array}$} \\
\hline & RA & RI & GR & RO & $\mathrm{AC}$ & \\
\hline 1 & 242 & - & 418 & 1321 & 275 & \\
\hline 2 & 251 & 1607 & 322 & 1277 & 279 & $72 \%$ \\
\hline 3 & 257 & 1412 & 349 & 1605 & 275 & $71 \%$ \\
\hline 4 & 237 & - & 299 & - & 279 & \\
\hline 5 & 234 & 1892 & 309 & - & 249 & \\
\hline 6 & 226 & 1844 & 278 & - & 280 & \\
\hline 7 & 237 & - & 274 & - & 255 & \\
\hline 8 & 223 & - & 251 & - & 255 & \\
\hline 9 & 218 & 1610 & 214 & - & 253 & \\
\hline 10 & 220 & 1538 & 211 & - & 248 & \\
\hline 11 & 218 & 1438 & 230 & - & 263 & \\
\hline 12 & 207 & 1365 & 232 & - & 239 & \\
\hline 13 & 222 & 1239 & 260 & - & 211 & \\
\hline 14 & 209 & 1325 & 238 & - & 214 & \\
\hline 15 & 237 & 1607 & 277 & - & 230 & \\
\hline 16 & 248 & - & 274 & - & 232 & \\
\hline 17 & 255 & - & 302 & - & 241 & \\
\hline 18 & 280 & - & 324 & - & 282 & \\
\hline 19 & 269 & - & 371 & - & 292 & \\
\hline 20 & 275 & - & 371 & - & 285 & \\
\hline Average generation & 238 & - & 290 & - & 257 & $54 \%$ \\
\hline
\end{tabular}

\subsection{Discussion for Assigning Gain $\alpha$ and Method}

We discuss the convergence of the optimization process using the trend of benchmark function with the difference from the calculation cost among models. As shown in Fig. 11$15, \mathrm{APGA} / \mathrm{VNC} 1$ converged earlier than other models in early generations, that because this model updated the elite individual with certainly. However, after that, APGA/VNC1 could not update it efficiently, and finally other models reach a global optimum solution faster than this one.

H-APGA1 was really good with RI function but it could not get high probability with RO function, compared with H-APGA2. H-APGA2 could reach a global optimum at a high probability with every function.

We made various tests using the values of gain $\alpha$ that ranges from 0 to 20 of $\mathrm{RO}$ function with 20 dimensions by H-APGA method. From this comparison as shown in Fig. 16, we confirmed that H-APGA2 converged faster than H-APGA1, and the best value of gain $\alpha$ is 10 . That is the reason why we assigned this value within all experiments.

To sum up, from these results via optimization experiments, we employed APGA/VNC2 for the APGA/VNC, and H-APGA2 for H-APGA. As shown in Fig. 17, H-APGA converged faster than $\mathrm{APGA} / \mathrm{VNC}$ with high improvement rate. 
Table 5 Experiment results by APGA/VNC2. Number of generations required to reach the global optimum (20 dimensions; population size 50)

\begin{tabular}{|c|c|c|c|c|c|c|}
\hline \multirow{2}{*}{ Trial } & \multicolumn{5}{|c|}{ Function } & \multirow{2}{*}{$\begin{array}{c}\text { Improvement } \\
\text { Rate }\end{array}$} \\
\hline & RA & RI & $\overline{\text { GR }}$ & $\mathrm{RO}$ & $\mathrm{AC}$ & \\
\hline 1 & 261 & 1264 & 361 & 1229 & 216 & $66 \%$ \\
\hline 2 & 246 & 1269 & 354 & 1025 & 216 & $65 \%$ \\
\hline 3 & 242 & 1620 & 292 & 962 & 260 & $69 \%$ \\
\hline 4 & 243 & 1481 & 302 & 1229 & 245 & $67 \%$ \\
\hline 5 & 228 & 1190 & 289 & 1025 & 243 & $61 \%$ \\
\hline 6 & 235 & 1366 & 288 & 962 & 254 & $65 \%$ \\
\hline 7 & 255 & 1090 & 295 & 827 & 226 & $59 \%$ \\
\hline 8 & 240 & 1071 & 281 & 997 & 257 & $61 \%$ \\
\hline 9 & 216 & 929 & 268 & 898 & 253 & $57 \%$ \\
\hline 10 & 226 & 1155 & 302 & 939 & 230 & $61 \%$ \\
\hline 11 & 227 & 1111 & 262 & 844 & 251 & $59 \%$ \\
\hline 12 & 241 & 1224 & 290 & 1000 & 238 & $62 \%$ \\
\hline 13 & 233 & 1306 & 314 & 1302 & 247 & $65 \%$ \\
\hline 14 & 229 & 1136 & 291 & 1092 & 250 & $61 \%$ \\
\hline 15 & 247 & 1096 & 310 & 906 & 278 & $65 \%$ \\
\hline 16 & 246 & 1153 & 340 & 1018 & 279 & $67 \%$ \\
\hline 17 & 251 & 1321 & 332 & 1008 & 294 & $70 \%$ \\
\hline 18 & 274 & 1151 & 379 & 1052 & 297 & $72 \%$ \\
\hline 19 & 269 & 1267 & 361 & 1175 & 307 & $73 \%$ \\
\hline 20 & 269 & 1476 & 426 & 1302 & 300 & $79 \%$ \\
\hline Average generation & 242 & 1234 & 317 & 1040 & 257 & $65 \%$ \\
\hline
\end{tabular}

Table 6 Experiment results by H-APGA1. Number of generations required to reach the global optimum (20 dimensions; population size 50)

\begin{tabular}{|c|c|c|c|c|c|c|}
\hline \multirow{2}{*}{ Trial } & \multicolumn{5}{|c|}{ Function } & Improvement \\
\cline { 2 - 6 } & RA & RI & GR & RO & AC & Rate \\
\hline 1 & 219 & 533 & 384 & 1792 & 84 & $47 \%$ \\
\hline 2 & 220 & 461 & 429 & 1456 & 169 & $54 \%$ \\
\hline 3 & 206 & 648 & 429 & 1779 & 107 & $52 \%$ \\
\hline 4 & 166 & 1218 & 377 & 1466 & 174 & $58 \%$ \\
\hline 5 & 218 & 469 & 305 & 1863 & 125 & $45 \%$ \\
\hline 6 & 216 & 1209 & 387 & 1664 & 104 & $57 \%$ \\
\hline 7 & 194 & 617 & 421 & 1693 & 98 & $49 \%$ \\
\hline 8 & 217 & 633 & 448 & 1479 & 100 & $53 \%$ \\
\hline 9 & 202 & 602 & 402 & 1800 & 175 & $54 \%$ \\
\hline 10 & 205 & 270 & 471 & 1072 & 119 & $50 \%$ \\
\hline 11 & 217 & 983 & 301 & 1825 & 116 & $50 \%$ \\
\hline 12 & 193 & 661 & 472 & 1851 & 93 & $52 \%$ \\
\hline 13 & 212 & 672 & 459 & 1118 & 107 & $54 \%$ \\
\hline 14 & 213 & 637 & 360 & 1574 & 124 & $49 \%$ \\
\hline 15 & 218 & 237 & 352 & 1873 & 113 & $44 \%$ \\
\hline 16 & 195 & 738 & 409 & 1612 & 151 & $54 \%$ \\
\hline 17 & 207 & 472 & 403 & 1810 & 155 & $51 \%$ \\
\hline 18 & 210 & 705 & 403 & 1303 & 139 & $53 \%$ \\
\hline $\mathbf{1 9}$ & $\mathbf{2 0 5}$ & $\mathbf{3 6 2}$ & $\mathbf{2 3 2}$ & $\mathbf{1 8 7 8}$ & $\mathbf{1 2 1}$ & $\mathbf{3 8 \%}$ \\
\hline 20 & 217 & 273 & 337 & 1443 & 138 & $45 \%$ \\
\hline Average generation & 208 & 620 & 390 & 1618 & 126 & $51 \%$ \\
\hline & & & & & \\
\hline
\end{tabular}


Table 7 Experiment results by H-APGA2. Number of generations required to reach the global optimum (20 dimensions; population size 50)

\begin{tabular}{|c|c|c|c|c|c|c|}
\hline \multirow{2}{*}{ Trial } & \multicolumn{5}{|c|}{ Function } & Improvement \\
\cline { 2 - 6 } & RA & RI & GR & RO & AC & Rate \\
\hline 1 & 209 & 1236 & 276 & 1049 & 193 & $57 \%$ \\
\hline 2 & 172 & 943 & 307 & 1108 & 182 & $51 \%$ \\
\hline 3 & 212 & 822 & 327 & 1162 & 231 & $57 \%$ \\
\hline 4 & 207 & 1189 & 274 & 835 & 225 & $58 \%$ \\
\hline 5 & 180 & 1759 & 305 & 1057 & 244 & $66 \%$ \\
\hline 6 & 200 & 1850 & 298 & 1184 & 190 & $65 \%$ \\
\hline 7 & 214 & 738 & 299 & 1134 & 232 & $55 \%$ \\
\hline 8 & 167 & 831 & 311 & 1171 & 227 & $53 \%$ \\
\hline 9 & 211 & 858 & 319 & 1016 & 195 & $55 \%$ \\
\hline 10 & 205 & 657 & 275 & 1212 & 267 & $54 \%$ \\
\hline 11 & 190 & 1256 & 323 & 1076 & 175 & $57 \%$ \\
\hline 12 & 207 & 1844 & 239 & 1061 & 217 & $64 \%$ \\
\hline 13 & 178 & 611 & 305 & 1045 & 194 & $48 \%$ \\
\hline 14 & 206 & 847 & 316 & 976 & 279 & $59 \%$ \\
\hline 15 & 201 & 605 & 311 & 1022 & 231 & $53 \%$ \\
\hline 16 & 205 & 1042 & 327 & 1191 & 172 & $55 \%$ \\
\hline $\mathbf{1 7}$ & $\mathbf{1 2 5}$ & $\mathbf{7 5 0}$ & $\mathbf{3 0 6}$ & $\mathbf{1 1 5 6}$ & $\mathbf{1 4 1}$ & $\mathbf{4 3 \%}$ \\
\hline 18 & 210 & 755 & 294 & 1116 & 262 & $56 \%$ \\
\hline 19 & 201 & 1664 & 242 & 1190 & 206 & $60 \%$ \\
\hline 20 & 208 & 1057 & 293 & 999 & 196 & $55 \%$ \\
\hline Average generation & 196 & 1066 & 298 & 1088 & 213 & $56 \%$ \\
\hline & & & & & \\
\hline
\end{tabular}

\section{-APGA/VNC1 $\backsim \mathrm{APGA} / \mathrm{VNC2} \triangle \mathrm{H}-\mathrm{APGA} 1 \times \mathrm{H}-\mathrm{APGA} 2$}

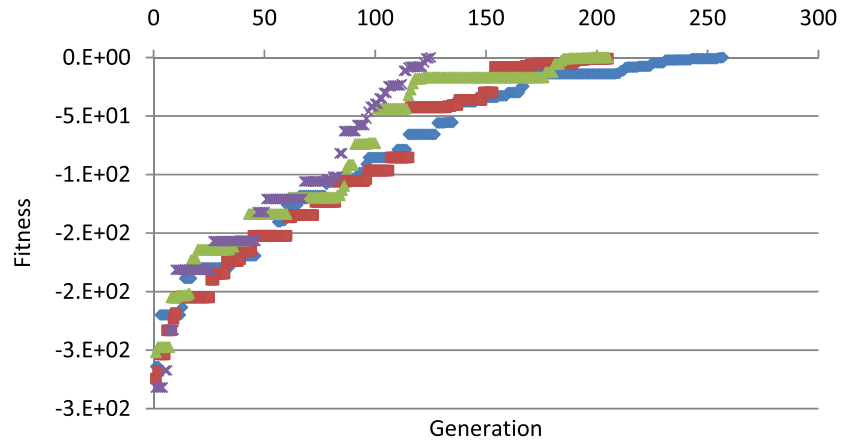

Fig. 11 Elite individual's fitness of RA function with 20 dimensions

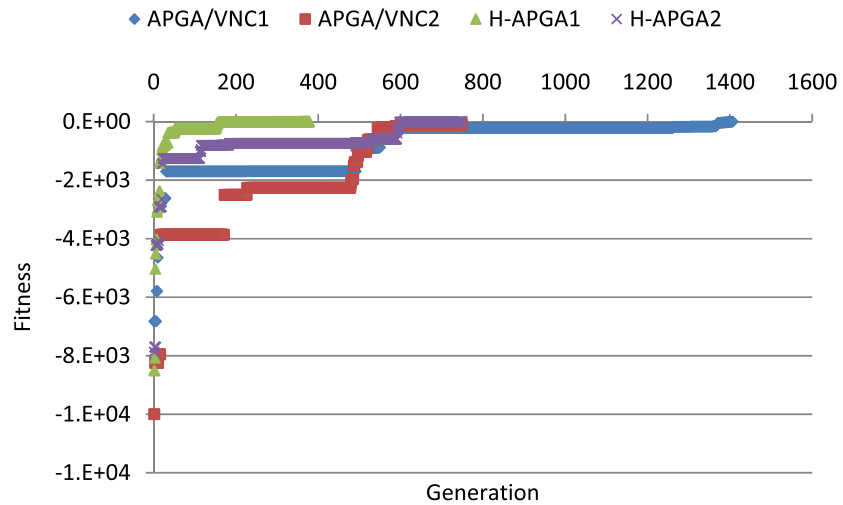

Fig. 12 Elite individual's fitness of RI function with 20 dimensions 


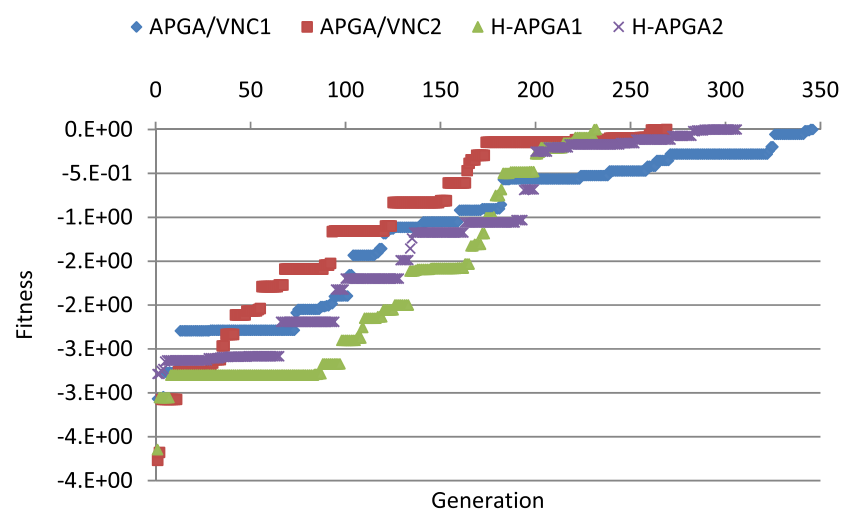

Fig. 13 Elite individual's fitness of GR function with 20 dimensions

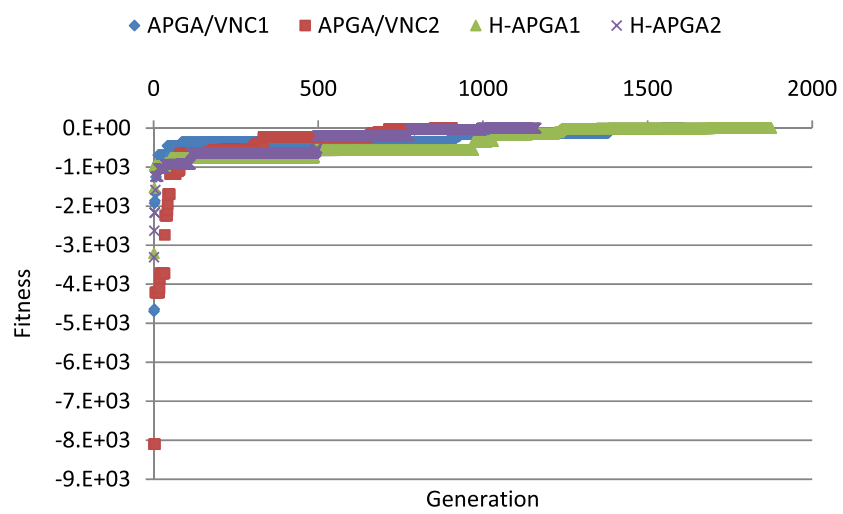

Fig. 14 Elite individual's fitness of RO function with 20 dimensions

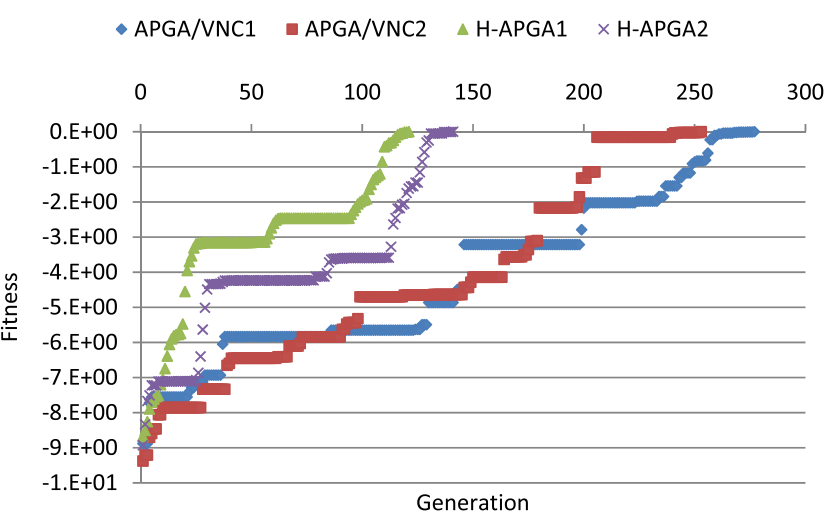

Fig. 15 Elite individual's fitness of AC function with 20 dimensions

\subsection{Comparison for Robustness}

All new evolutionary strategies of APGA (APGAs) - simple APGA ${ }^{(25)}$, APGA/VNC (26) , and H-APGA ${ }^{(27)}$ were compared with other methodologies - Parallel SA with Selection (PSAS) in Ref. (14), Hybrid meta-heuristics (HMH) in Ref. (17), (24). The comparison is given in Table 8.

By means of the comparison lists, APGAs could certainly achieve optimal solution with low calculation cost. The convergence of the optimal solution could be improved more significantly in APGAs than that in EAs for the same calculation cost.

H-APGA had better convergence than APGA/VNC with four benchmark functions RA, RI, GR and AC. However, H-APGA did not gain a high probability with RO function as $\mathrm{APGA} / \mathrm{VNC}$.

The comparison among all strategies of APGA is also shown in Fig. 18. APGA/VNC and H-APGA were better than simple APGA in all benchmark functions. Therefore, it is desirable to introduce these models into APGA. 


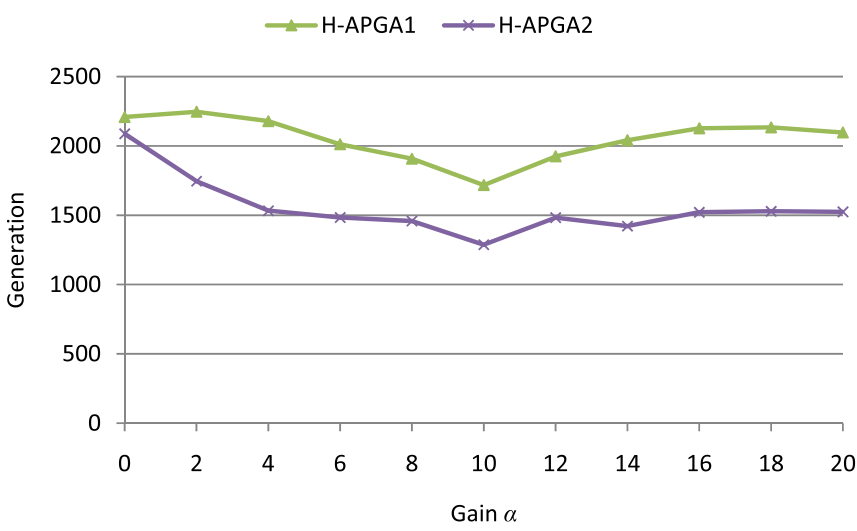

Fig. 16 Gain $\alpha$ by H-APGA method of RO function with 20 dimensions

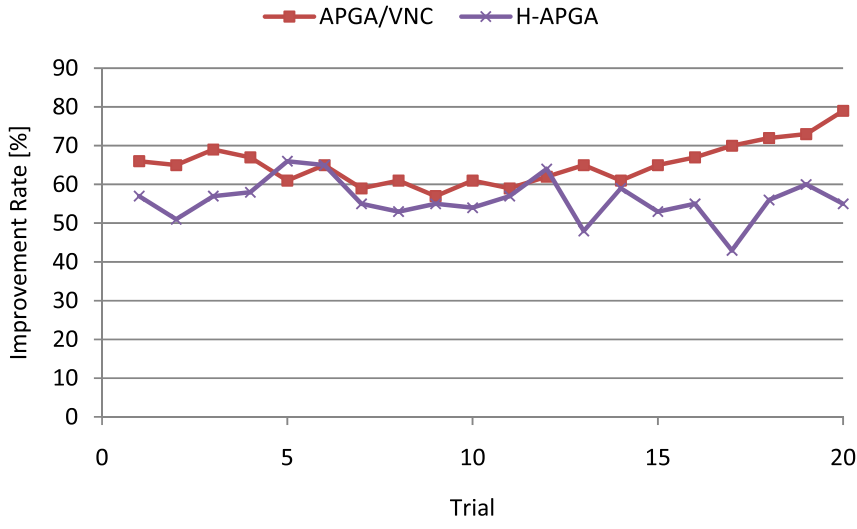

Fig. 17 The improvement rate through trials

Table 8 Average generations over 20 independent trials by PSAS, HMH, APGA, APGA/VNC and H-APGA (20 dimensions; population size 50)

\begin{tabular}{|c|c|c|c|c|c|c|c|}
\hline \multirow{2}{*}{ Function } & \multirow{2}{*}{ Dim. } & & $\begin{array}{c}\text { PSAS } \\
\text { Ref. (14) }\end{array}$ & $\begin{array}{c}\text { HMH } \\
\text { Ref. (17), (24) }\end{array}$ & $\begin{array}{c}\text { APGA } \\
\text { Ref. (25) }\end{array}$ & $\begin{array}{c}\text { APGA/VNC } \\
\text { Ref. (26) }\end{array}$ & $\begin{array}{c}\text { H-APGA } \\
\text { Ref. (27) }\end{array}$ \\
\hline \multirow{2}{*}{ RA } & \multirow{2}{*}{20} & Generation & 1,500 & 3,630 & 376 & 242 & 196 \\
\cline { 3 - 8 } & & Func. call & 150,000 & 145,184 & 18,800 & 12,100 & 9,800 \\
\hline \multirow{2}{*}{ RI } & \multirow{2}{*}{20} & Generation & 5,200 & 17,686 & 2,030 & 1234 & 1066 \\
\cline { 3 - 8 } & & Func. call & 520,000 & 707,440 & 101,500 & 61,700 & 53,300 \\
\hline \multirow{2}{*}{ GR } & \multirow{2}{*}{20} & Generation & 4,100 & 9,003 & 468 & 317 & 298 \\
\cline { 3 - 8 } & & Func. call & 410,000 & 360,132 & 23,400 & 15,850 & 14,900 \\
\hline \multirow{2}{*}{ RO } & \multirow{2}{*}{20} & Generation & - & - & - & 1,040 & 1088 \\
\cline { 3 - 8 } & & Func. call & - & - & - & 52,000 & 54,400 \\
\hline \multirow{2}{*}{ AC } & \multirow{2}{*}{20} & Generation & 1,500 & 3,309 & 378 & 257 & 213 \\
\cline { 3 - 8 } & & Func. call & 150,000 & 132,360 & 18,900 & 12,850 & 10,650 \\
\hline
\end{tabular}

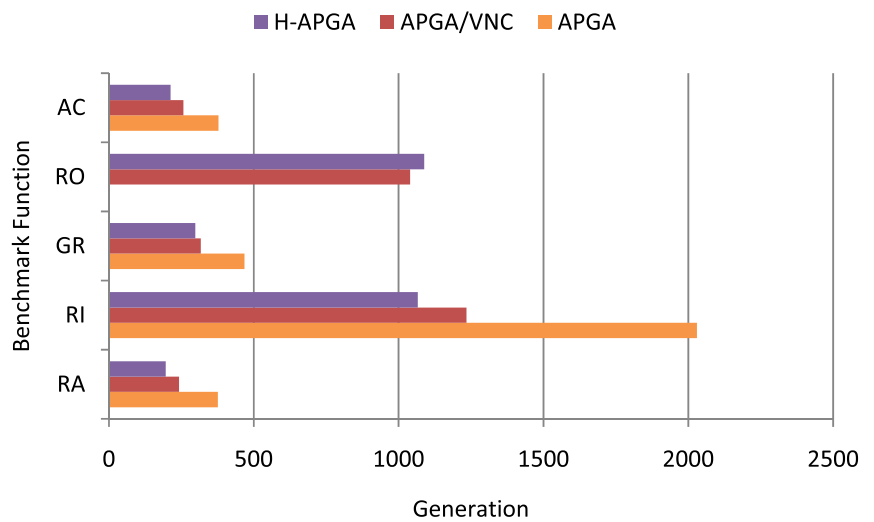

Fig. 18 Comparison of APGA, APGA/VNC and H-APGA 
To evaluate the performance of APGAs, we compared to other EAs such as GA, Particle Swarm optimization (PSO), Particle Swarm Inspired Evolutionary Algorithm (PS-EA) in Ref. (28) and Artificial Bee Colony $(\mathrm{ABC})^{(29)}$, Differential Evolution (DE) ${ }^{(30)}$ and SelfAdaptive DE $(\mathrm{SaDE})^{(31)}$, and Advanced DE $(\mathrm{ADE})^{(32)}$. Maximum number of generation and the population size, i.e. 100, 125, as in the study presented in Ref. (28), (31). The mean and the standard deviations of the function values obtained by these methods are given in Table 9 and Table 10.

In particular, it confirmed that the calculation cost with these models could be reduced for benchmark functions. And it showed that the convergence to the optimal solution could be significantly improved.

The advantage of the APGAs over other EAs seems to be limited because the APGAs specify small number of digits of design variables in the numerical experiments.

Overall, APGAs was capable of attaining robustness, high quality, low calculation cost and efficient performance on many benchmark problems. We confirmed satisfactory performance through various benchmark tests.

Table 9 The mean and the standard deviations obtained by GA, PSO, PS-EA, ABC and APGAs (30 dimensions; population size 125)

\begin{tabular}{|c|c|c|c|c|c|c|c|}
\hline Function & Dim. & Gen. & $\begin{array}{c}\mathrm{GA}^{(28)} \\
\text { Mean } \\
(\text { Std Dev })\end{array}$ & $\begin{array}{c}\text { PSO }^{(28)} \\
\text { Mean } \\
(\text { Std Dev })\end{array}$ & $\begin{array}{c}\text { PS-EA }^{(28)} \\
\text { Mean } \\
(\text { Std Dev })\end{array}$ & $\begin{array}{c}\text { ABC } \\
\text { Mean } \\
(\text { Std Dev })\end{array}$ & $\begin{array}{c}\text { APGAs } \\
\text { Mean } \\
(\text { Std Dev })\end{array}$ \\
\hline RA & 30 & 1000 & $\begin{array}{c}10.4388 \\
(2.6386)\end{array}$ & $\begin{array}{c}32.476 \\
(6.9521)\end{array}$ & $\begin{array}{c}3.0527 \\
(0.9985)\end{array}$ & $\begin{array}{c}0.033874 \\
(0.181557)\end{array}$ & $\begin{array}{c}0 \\
(0)\end{array}$ \\
\hline RI & 30 & 1000 & - & - & - & - & $\begin{array}{c}0 \\
(0)\end{array}$ \\
\hline GR & 30 & 1000 & $\begin{array}{c}1.2342 \\
(0.11045)\end{array}$ & $\begin{array}{c}0.011151 \\
(0.014209)\end{array}$ & $\begin{array}{c}0.8211 \\
(0.1394)\end{array}$ & $\begin{array}{c}2.87 \mathrm{E}-09 \\
(8.45 \mathrm{E}-10)\end{array}$ & $\begin{array}{c}0 \\
(0)\end{array}$ \\
\hline RO & 30 & 1000 & $\begin{array}{c}166.283 \\
(59.5102)\end{array}$ & $\begin{array}{c}402.54 \\
(633.65)\end{array}$ & $\begin{array}{c}98.407 \\
(35.5791)\end{array}$ & $\begin{array}{c}0.219626 \\
(0.152742)\end{array}$ & $\begin{array}{c}-1.29 \mathrm{E}-02 \\
(1.03 \mathrm{E}-02)\end{array}$ \\
\hline AC & 30 & 1000 & $\begin{array}{c}1.0989 \\
(0.24956)\end{array}$ & $\begin{array}{c}1.49 \mathrm{E}-06 \\
(1.86 \mathrm{E}-06)\end{array}$ & $\begin{array}{c}0.3771 \\
(0.098762)\end{array}$ & $\begin{array}{c}3.00 \mathrm{E}-12 \\
(5.00 \mathrm{E}-12)\end{array}$ & $\begin{array}{c}-4.44 \mathrm{E}-16 \\
(0.00 \mathrm{E}+00)\end{array}$ \\
\hline
\end{tabular}

Table 10 The mean and the standard deviations obtained by DE, SaDE, ADE and APGAs (30 dimensions; population size 100)

\begin{tabular}{|c|c|c|c|c|c|c|}
\hline Function & Dim. & Gen. & $\begin{array}{c}\mathrm{DE}^{(30)} \\
\text { Mean } \\
\text { (Std Dev) }\end{array}$ & $\begin{array}{c}\mathrm{SaDE}^{(3 \mathrm{I})} \\
\text { Mean } \\
(\mathrm{Std} \text { Dev) }\end{array}$ & $\begin{array}{c}\mathrm{ADE}^{(32)} \\
\text { Mean } \\
\text { (Std Dev) }\end{array}$ & $\begin{array}{c}\text { APGAs } \\
\text { Mean } \\
\text { (Std Dev) }\end{array}$ \\
\hline RA & 30 & 1500 & $\begin{array}{l}173.405 \\
(13.841)\end{array}$ & $\begin{array}{c}1.50 \mathrm{E}-15 \\
(4.80 \mathrm{E}-15)\end{array}$ & $\begin{array}{c}0 \\
(0)\end{array}$ & $\begin{array}{c}0 \\
(0)\end{array}$ \\
\hline RI & 30 & 1500 & $\begin{array}{c}1.63086 \\
(0.886153)\end{array}$ & $\begin{array}{c}0.090075 \\
(0.080178)\end{array}$ & - & $\begin{array}{c}0 \\
(0)\end{array}$ \\
\hline GR & 30 & 1500 & $\begin{array}{c}2.90 \mathrm{E}-13 \\
(4.20 \mathrm{E}-13)\end{array}$ & $\begin{array}{c}0 \\
(0)\end{array}$ & $\begin{array}{c}0 \\
(0)\end{array}$ & $\begin{array}{c}0 \\
(0)\end{array}$ \\
\hline RO & 30 & 1500 & $\begin{array}{c}7.80 \mathrm{E}-09 \\
(5.80 \mathrm{E}-09)\end{array}$ & $\begin{array}{c}3.10 \mathrm{E}-15 \\
(8.30 \mathrm{E}-15)\end{array}$ & $\begin{array}{c}3.57 \mathrm{E}-05 \\
(8.90 \mathrm{E}-05)\end{array}$ & $\begin{array}{c}0 \\
(0)\end{array}$ \\
\hline $\mathrm{AC}$ & 30 & 1500 & $\begin{array}{c}9.70 \mathrm{E}-08 \\
(4.20 \mathrm{E}-08)\end{array}$ & $\begin{array}{c}7.70 \mathrm{E}-15 \\
(1.40 \mathrm{E}-15)\end{array}$ & $\begin{array}{c}2.66 \mathrm{E}-15 \\
(0.00 \mathrm{E}+00)\end{array}$ & $\begin{array}{c}-4.44 \mathrm{E}-16 \\
(0.00 \mathrm{E}+00)\end{array}$ \\
\hline
\end{tabular}

\subsection{APGAs with 100 Dimensions and Different Population}

We applied to benchmark tests with 100 dimensions by 20 independent runs and $10,000^{\text {th }}$ terminal generation. APGA/VNC and H-APGA could reach global optimum solution with all benchmark functions, and the success rate of optimal soluiton is $100 \%$. The experiment results, average generations required to reach the global optimum are given in Table 11.

From this table, it can be concluded that as the population size increases, the performance of the APGAs significantly improves. Additionally, the results show that the proposed APGAs is effective in all benchmarks for various population sizes. The performance of the APGAs show relatively improvement with the growth of the population size, which suggests that the APGAs is more stable and robust on population size. 
Table 11 Experimental results, averaged over 20 runs by APGAs of 100 dimesions with different population size. "Mean" indicates average of optimum values obtained and "Std Dev" stands for standard deviation

\begin{tabular}{|c|c|c|c|}
\hline \multicolumn{4}{|c|}{ Population size 50 } \\
\hline Function & APGA/VNC & H-APGA & Mean (Std Dev) \\
\hline RA & 737 & 729 & $0.00 \mathrm{E}+00(0.00 \mathrm{E}+00)$ \\
\hline RI & 2736 & 3777 & $0.00 \mathrm{E}+00(0.00 \mathrm{E}+00)$ \\
\hline GR & 1464 & 1230 & $0.00 \mathrm{E}+00(0.00 \mathrm{E}+00)$ \\
\hline RO & 5430 & 5514 & $0.00 \mathrm{E}+00(0.00 \mathrm{E}+00)$ \\
\hline AC & 828 & 644 & $-4.44 \mathrm{E}-16(0.00 \mathrm{E}+00)$ \\
\hline \multicolumn{4}{|c|}{ Population size 100} \\
\hline Function & APGA/VNC & H-APGA & Mean (Std Dev) \\
\hline RA & 592 & 540 & $0.00 \mathrm{E}+00(0.00 \mathrm{E}+00)$ \\
\hline RI & 1145 & 1579 & $0.00 \mathrm{E}+00(0.00 \mathrm{E}+00)$ \\
\hline GR & 980 & 904 & $0.00 \mathrm{E}+00(0.00 \mathrm{E}+00)$ \\
\hline RO & 2819 & 2802 & $0.00 \mathrm{E}+00(0.00 \mathrm{E}+00)$ \\
\hline AC & 432 & 371 & $-4.44 \mathrm{E}-16(0.00 \mathrm{E}+00)$ \\
\hline
\end{tabular}

\section{Conclusion}

In this paper, to overcome the weak point of EAs, and to achieve the global search for the solution space of multi-peak optimization problems with multi-dimensions, we proposed new evolution strategies of Adaptive Plan System with Genetic Algorithm, APGAs. Then, we verified the effectiveness of APGAs by the numerical optimization experiments performed five benchmark functions. The obtained points are shown below

The search ability of APGAs with multi-dimensions optimization problems is very effective, compared with that of EAs. Nevertheless, the number of digits of design variables in the numerical experiments is insufficient to discuss about the stability of convergence.

All the strategies of APGA achieved at the global optimum solution. However, these models have strengths and weaknesses. About the optimal solution such as minimum time and maximum reliability, it is a future work.

Finally, this study plans to do a comparison with the sensitivity plan of AP by applying other optimization methods into AP and optimizing benchmark tests, constrained realparameters, futher real life applications.

\section{References}

( 1 ) Holland, J., Adaptation in Natural and Artificial Systems, The University of Michigan 1975, MIT Press, (1992).

( 2 ) Goldberg, D.E., Genetic Algorithms in Search Optimization and Machine Learning, (1989), Addison- Wesley.

( 3 ) Digalakis, J.D. and Margaritis, K.G., An experimental study of benchmarking functions for Genetic Algorithms, Proceedings of IEEE Conference on Transactions, Vol.5, (2000), pp.3810-3815.

( 4 ) Sakuma, J. and Kobayashi, S., Extrapolation-Directed Crossover for Real-coded GA: Overcoming Deceptive Phenomena by Extrapolative Search, Proceedings of Congress on Evolutionary Computation (CEC 2001), (2001), pp.685-662.

( 5 ) Li, X. and Kirley, M., The Effects of Varying Population Density in a Fine-grained Parallel Genetic Algorithm, Proceedings of Congress on Evolutionary Computation (CEC 2002), No.2, (2002), pp.1709-1714.

( 6 ) Hasegawa, H. and Kawamo, K., A Method for the Shape and Topology Optimization of Mechanical Structures by Using Genetic Algorithm (Layout Optimization Method by Adoption of Removal and Addition parameters of Elements as Chromosomes), Transactions of the Japan Society of Mechanical Engineers, Series A, Vol.61, No.581 (1995), pp.183-190 (in Japanese).

( 7 ) Tsuruta, Y., Hasegawa, H. and Kawamo, K., A Method for the Shape and Topology Optimization of Mechanical Structures Using Genetic Algorithm (2nd Report, On Con- 
vergence of Solutions of Our Method by Adoption of Removal and Addition parameters of Elements as Chromosomes), Transactions of the Japan Society of Mechanical Engineers, Series A, Vol.63, No.605, (1997), pp.170-177 (in Japanese).

( 8 ) Tsuruta, Y., Suzuki, Y., Hasegawa, H. and Kawamo, K., A Method for the Shape and Topology Optimization of Mechanical Structures Using Genetic Algorithm, JSME Centenial Grand Congress by the JSME International Symposium on OPID97, (1997), Paper 137.

( 9 ) Nishi, H., Okamoto, T. and Kawamo, K., Study on the Development of General-purpose Genetic Algorithm Engine, Extend Abstracts of WCSMO-4, (2001), pp.428-429.

(10) Kawada, H., Uehara, H. and Kawamo, K., Development of Generalpurposed Optimization Engine and Test Run, Journal of Japan Society for Simulation Technology, Vol.21, No.4, (2003), pp.280-288 (in Japanese).

(11) Rosen, B.E. and Nakano, R., Simulated annealing - basics and recent topics on simulated annealing, Journal of Japanese Society for Artificial Intelligence, Vol.9, No.3, (1994) (in Japanese).

(12) Mahfoud, S.W. and Goldberg, D.E., A genetic algorithm for parallel simulated annealing, Parallel Problem Solving from Nature, No.2, (1992), pp.301-310.

(13) Uehara, H., Kawada, H. and Kawamo, K., Numerical Experiments on Optimal Points Searching Using Hybrid Method of Genetic Algorithm and Simulated Annealing, 52nd Japan National Congress for Theoretical and Applied Mechanics, (2003), pp.117-118 (in Japanese).

(14) Uehara, H., Study on Development of General-purposed Optimization Engine and its Performance Evaluation - The proposal of Parallel Simulated Annealing with Selection, $M$ of Eng thesis, Graduate School of Engineering, Shibaura Institute of Technology, (2004) (in Japanese).

(15) Hiroyasu, T., Miki, M. and Ogura, M., Parallel Simulated Annealing using Genetic Crossover, Proceedings of the ISCA 13th International Conference on PDCS-2000, (2000).

(16) Miki, M., Hiroyasu, T. and Fushimi, T., Parallel Simulated Annealing with Adaptive Neighborhood Determined by GA, IEEE International Conference on System, Man and Cybernetics, (2003).

(17) Hasegawa, H., Yoshikawa, M., Uehara, H. and Kawamo, K., The Hybrid Meta-heuristics by Reflecting Recognition of Dependence Relation among Design Variables for Integer Optimization of Multi-peak Problems, Journal of Japan Society for Simulation Technology, Vol.25, No.2, (2006), pp.144-155 (in Japanese).

(18) Ong, Y.S. and Keane, A.J., Meta-Lamarckian Learning in Memetic Algorithms, IEEE transactions on evolutionary computation, Vol.8, No.2, (2004), pp.99-110.

(19) Hart, W.E., Krasnogor, N. and Smith, J.E., Recent Advances in Memetic Algorithms, (2005), Springer.

(20) Ong, Y.S., Lim, M.H., Zhu, N. and Wong, K.W., Classification of Adaptive Memetic Algorithms: A Comparative Study, IEEE transactions on Systems, Man and Cybnetics Part B, Vol.36, No.1, (2006), pp.141-152.

(21) Neri, F., Tirronen, V., Kärkkäinen, T. and Rossi, T., Fitness Diversity Based Adaptation in Multimeme Algorithms: A Comparative Study, IEEE Congress on Evolutionary Computation (CEC 2007), (2007), pp.2374-2381.

(22) Caponio, A., Cascella, G.L., Neri, F., Salvatore, N. and Sumner,M., A Fast Adaptive Memetic Algorithm for Online and Offline Control Design of PMSM Drives, IEEE transactions on Systems, Man and Cybernetics Part B, Special Issue on Memetic Algorithms, Vol.37, No.1, (2007), pp.28-41.

(23) Tirronen, V., Neri, F., Kärkkäinen, T., Majava, K. and Rossi, T., An Enhanced Memetic Differential Evolution in Filter Design for Defect Detection in Paper Production, Evolutionary Computation Journal, MIT Press, Vol.16, No.4, (2008), pp.529-555. 
(24) Hasegawa, H., Sasaki, H., Uehara, H. and Kawamo, K., The optimisation of spot-weld positions for vehicle design by using hybrid metaheuristics, International Journal of Vehicle Design, Vol.43, No.1-4, (2007), pp.151-172.

(25) Hasegawa, H., Adaptive Plan System with Genetic Algorithm based on Synthesis of Local and Global Search Method for Multi-Peak Optimization Problems, Proceedings of the 6th EUROSIM Congress on Modelling and Simulation, (2007).

(26) Tooyama, S. and Hasegawa, H., Adaptive Plan System with Genetic Algorithm using the Variable Neighborhood Range Control, IEEE Congress on Evolutionary Computation (CEC 2009), (2009), pp.846-853.

(27) Pham, N.H., and Hasegawa, H., Hybrid neighborhood control method of adaptive plan system with genetic algorithm, Proceeding of the 7th EUROSIM Congress on Modelling and Simulation, (2010).

(28) Srinivasan, D., and Seow, T.H., Evolutionary Computation, IEEE Congress on Evolutionary Computation (CEC 2003), (2003), pp.2292-2297.

(29) Karaboga, D., and Basturk, B., A powerful and efficient algorithm for numerical function optimization: artificial bee colony (ABC) algorithm, Journal Global Optimization, Vol.39, (2006), pp.459-471.

(30) Storn, R., and Price, K., Differential Evolution - a simple and efficient Heuristic for global optimization over continuous spaces, Journal Global Optimization, Vol.11, No.4, (1997), pp.341-357.

(31) Brest, J., Greiner, S., Boskovic, B., Mernik, M., and Zumer, V., Self-adapting control parameters in differential evolution: A comparative study on numerical benchmark problems, IEEE Transactions on Evolutionary Computation, Vol.10, No.6, (2006), pp.646657.

(32) Wagdy Mohamed, A., Sabry, H.Z., Farhat, A., Advanced Differential Evolution algorithm for global numerical optimization, IEEE International Conference on Computer Applications and Industrial Electronics (ICCAIE), (2011), pp.156-161. 